\title{
PASSERIFORMES DEL NEÓGENO IBÉRICO. HALLAZGOS EN LAYNA (PLIOCENO INFERIOR)
}

\author{
Antonio SÁNCHEZ', Benigno PÉREZ' y Susana HERRERO² \\ ' Departamento de Paleobiología. Museo Nacional de Ciencias Naturales. \\ c/. José Gutiérrez Abascal, 2. 28006 Madrid. \\ 2Departamento de Paleontología. Facultad de Ciencias Geológicas. Universidad Complutense. \\ 28040 Madrid.
}

\begin{abstract}
Sánchez, A., Pérez, B. y Herrero, S. 2000. Passeriformes del Neógeno ibérico. Hallazgos en Layna (Plioceno Inferior). [Passeriformes from the Iberian Neogene. Findings in Layna (Early Pliocene).] Revista Española de Paleontología, 15(1), 101-104. ISSN 0213-6937.
\end{abstract}

\begin{abstract}
Passeriforms constitute one of the spreadest and rich in species avian groups now a days. Such a condition was probably reached during the Miocene. However, their evolutionary history is also the worst known among the major orders of birds. There are a taphonomic ground to explain this fact, though the main difficulty consists on the scarce anatomical variation within the group, that complicates the classification and taxonomic identifications. We mention the findings at Layna site and the Tertiary fossil material kown in Iberia.
\end{abstract}

Keywords: Fossil birds, Layna, Early Pliocene, Iberian peninsula.

\section{RESUMEN}

Las Passeriformes constituyen uno de los grupos avianos de más amplia repartición y ricos en especies en la actualidad, condición que probablemente alcanzaron en el Mioceno. Sin embargo, su historia evolutiva es también la peor conocida de los grandes órdenes. Hay razones tafonómicas, pero la causa principal es la escasa variación anatómica en el grupo, lo que complica la clasificación y las identificaciónes taxonómicas. Se mencionan los restos encontrados en Layna, así como los conocidos hasta ahora en el Terciario ibérico.

Palabras clave: Aves fósiles, Layna, Plioceno inferior, Península Ibérica.

\section{INTRODUCCIÓN}

El yacimiento de Layna (Soria) está situado en el cerro Pelado, a unos $500 \mathrm{~m}$ del pueblo que da nombre al yacimiento. Está constituido por un relleno kárstico en las carniolas del límite Triásico-Jurásico, que forma la superficie estructural de Molina de Aragón. En la actualidad sólo quedan vestigios de las formas cársticas primitivas (Hoyos et al., 1974).

Desde su descubrimiento en el año 1964, este sitio paleontológico despertó un gran interés, tanto por la magnífica representación faunística (anfibios, reptiles, aves, micro y macromamíferos), como por su naturaleza kárstica. Se han reconocido diecisiete especies de macromamíferos, dos de las cuales son especies descritas por primera vez en este yacimiento (Pérez y Soria, 1990).

La primera excavación se realizó en 1972 bajo la dirección de E. Aguirre. En el año 1982 se llevaron a cabo nuevos trabajos dirigidos por M.T. Alberdi. A pesar del gran interés que suscitó este yacimiento en la década de los setenta y principios de los ochenta, así como del gran número de estudios a él dedicados, prácticamente ha caído en el olvido, sin que se hayan solucionado los problemas e interrogantes abiertos. Por este motivo, en agosto de 1998, se inició una serie de campañas dirigidas por B. Pérez.

Las Passeriformes son aves emperchadoras (arborícolas o arbustivas), de pequeña talla, con algunas familias que exhiben formas de vida terrestres. Las especies de mayor tamaño se encuentran en este último grupo adaptativo. La condición primitiva es la emperchadora, como muestran las tres trócleas digitales del tarsometatarso, situadas en un mismo plano y alineadas distalmente, configuración que se mantiene en todas las especies.

Las dificultades que presenta la identificación de las Passeriformes, incluso en el nivel familia, han determinado que éste sea uno de los grupos más desconocidos de los que poblaron Europa durante el Terciario. Una vez rechazada la pertenencia a este grupo de Protornis glarniensis Meyer y $P$. 

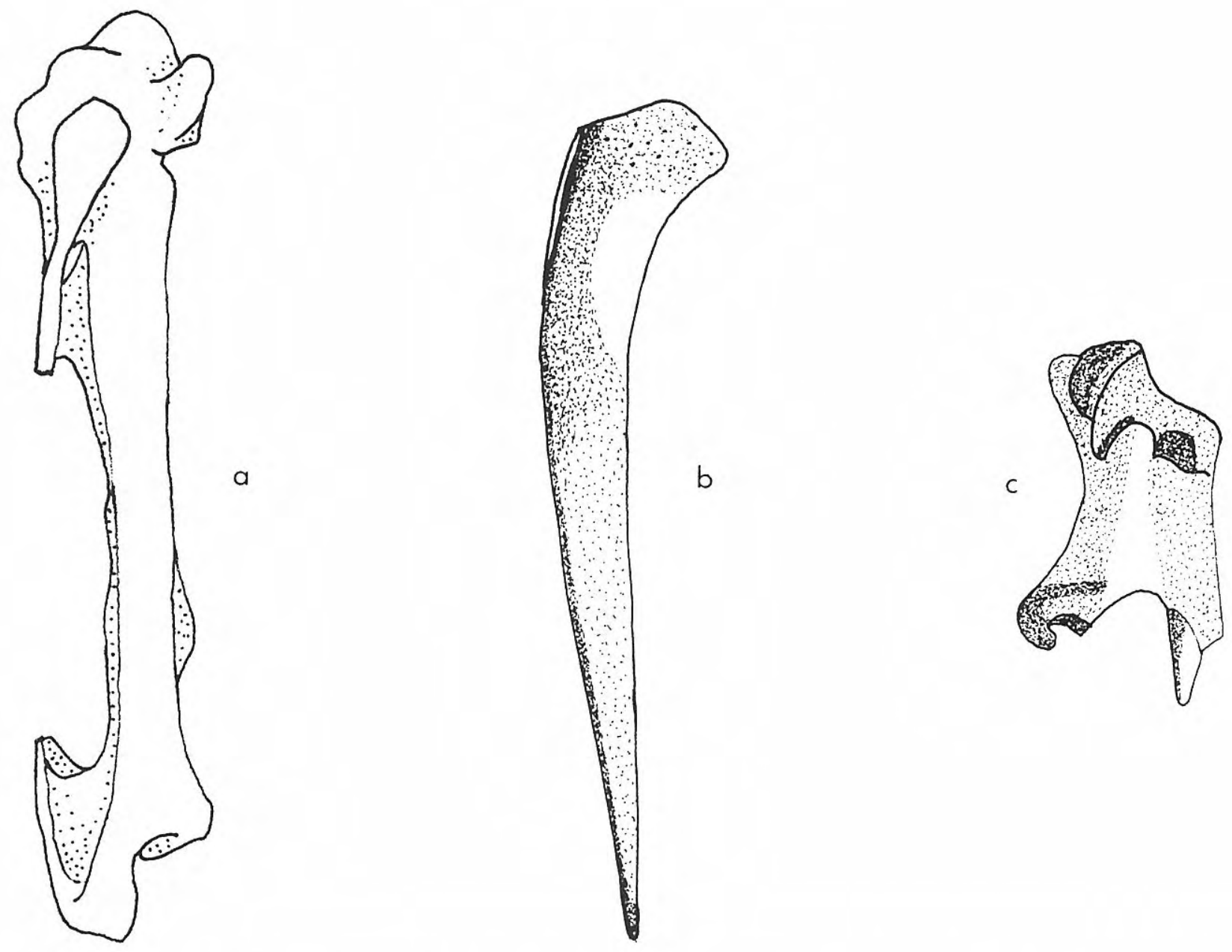

Figura 1. Tres restos de Passeriformes de Layna; colección del Museo Nacional de Ciencias Naturales (aumentados por ocho): a. carpometacarpo izquierdo (vista interna), b. fragmento de escápula (vista lateral), c. vértebra (vista lateral).

blumeri Heer, del yacimiento suizo de Matt, Oligoceno inferior (Olson, 1976; Mlíkovský, 1992), los registros más antiguos de Passeriformes en el hemisferio norte corresponden a tres localidades francesas del Oligoceno superior. Hay varios huesos identificados como "oscines indet." en Coderet y Gannat (Mourer-Chauviré et al., 1989), ambos en el departamento de Allier, biozona MP 30 (Schmidt-Kittler, 1987). Los géneros Turdus y Sitta han sido encontrados en Aix-en-Provence, también en la biozona MP 30 ( $c f$. Mourer-Chauviré, 1995). Probablemente, estos datos señalen la cronología de la aparición de las oscinas en Europa y no encontremos en el futuro representantes de este grupo antes del Oligoceno (Harrison, 1979; Olson, 1985; Mlíkovský, 1995). Pero no sería hasta el Mioceno cuando las Passeriformes alcanzarían una presencia dominante en el hemisferio norte, desplazando de los hábitats arbóreos a los Coraciiformes. Feduccia y Olson (1982) han sugerido que el origen de los paseriformes podría estar en el hemisferio sur, de donde no habrían salido hasta mediado el Terciario.

Las Passeriformes son numerosas en muchas localidades miocenas del hemisferio norte. El éxito de estas aves se detecta ya en el Mioceno inferior, como en Saint Gérand le Puy, departamento de Allier, biozona MN 2a ( $c f$. Cheneval,
1995). En el yacimiento de Wintershof (West), Baviera, biozona MN 3 (Kretzoi, 1985), los restos de los paseriformes suman más de la mitad del total de las aves.

\section{PASSERIFORMES EN LA PENÍNSULA IBÉRICA}

\section{Mioceno}

No hay noticia de paseriformes en los yacimientos portugueses del Terciario (Sánchez Marco, 1995a). Los más antiguos de la Península (cf. Sánchez Marco, 1995b) proceden del Vindoboniense de Hostalets de Piérola (Villalta y Crusafont, 1950), donde Villalta (1963) apunta la presencia de Fringilla a partir de la mitad distal de un húmero. Este fragmento óseo no tiene características diferenciales respecto de muchas de las familias del orden, por lo que una identificación aceptable sería: Passeriformes indet. En el yacimiento-trampa del Vallesiense de Batallones, Mioceno superior, MN 10 (Morales et al., 1992), existe un resto de un paseriforme del tamaño de un córvido pequeño (Sánchez Marco, en prensa) y otros huesos de especies de talla menor. 


\section{Plioceno}

En el Arquillo III, MN 15, se ha indicado Turdidae indet. (Mourer-Chauviré in Adrover et al., 1978). En el Cerro de los Espejos, MN 16a, se menciona el hallazgo de fósiles pertenecientes a Passeriformes indeterminadas (Adrover, 1975, 1986). Las Higueruelas, MN 16, cuenta con cinco familias Passeriformes: Motacillidae, Muscicapidae, Emberizidae, Ploceidae y Corvidae (Sánchez Marco, en preparación). Este es el yacimiento ibérico donde se constata por primera vez el predominio de este grupo de aves sobre las demás.

\section{Layna}

$\mathrm{Al}$ igual que el Arquillo III, Layna ha sido ubicada en la biozona MN 15 (Bruijn et al., 1992). Esta localidad ha ofrecido anteriormente interesantes hallazgos avianos. Es la localidad de donde se describió Chlamydotis mesetaria Sánchez Marco, 1990, y también se encontraron aquí restos de Tyto balearica, paleoespecie relacionada con la lechuza común actual (Mourer-Chauviré y Sánchez Marco, 1988). Recientemente, han aparecido cuatro huesos de passeriforme: un carpometacarpo (Fig. 1a), un fragmento de escápula (Fig. 1b), una vértebra (Fig. 1c) y una falange pedal. La morfología ósea de las Passeriformes es claramente diferente de la de los otros órdenes avianos. $\mathrm{Al}$ mismo tiempo, los rasgos distintivos entre las categorías de nivel inferior son sutiles, cuando las hay. La casi totalidad de estas diferencias morfológicas se observan en cráneo y húmero, elementos que aún no han sido hallados en Layna. Las medidas de los fósiles que ahora se reportan entran en el campo de variación de muchas familias, por lo que se puede afirmar que los restos pertenecen a passeriformes de pequeña talla, pero no es posible una identificación taxonómica precisa.

\section{INTERÉS DE LOS HALLAZGOS}

El predominio del orden de las Passeriformes, tanto en número de individuos como de especies, es una característica de la región holártica durante el Cuaternario. La amplia variedad de especies que este grupo de aves posee en la actualidad tiene un antecedente en la riqueza taxonómica que ha quedado registrada en el Mioceno inferior de Francia y Alemania. Durante el Plioceno, se efectuaron las últimas etapas del proceso de cambio faunístico que acabó configurando las ornitocenosis tal como las conocemos hoy. Hace entre 3 y 4 Ma, las ornitocenosis ibéricas habían adquirido ya un acusado carácter "cuaternario": había disminuido la proporción de aves terrestres, del tipo de Galliformes y Otididae, y se había producido un incremento de la proporción de Passeriformes.

La posición cronológica del yacimiento de Layna y la abundancia de especímenes de pequeña talla comienzan a proporcionar datos para perfilar los últimos cambios zoogeográficos y taxonómicos que tuvieron lugar durante la transición entre las ornitofaunas terciarias y las cuaternarias.

\section{AGRADECIMIENTOS}

Este trabajo se ha realizado con el apoyo económico de la Consejería de Educación y Cultura de la Junta de Castilla y León, proyecto: "Excavaciones paleontológicas en Cerro Pelado, Layna (Soria)”, subvención número: SO-03/1998.

\section{BIBLIOGRAFÍA}

Adrover, R. 1975. Principales yacimientos paleomastológicos de la provincia de Teruel y su posición estratigráfica relativa. Trabajos del Neógeno-Cuaternario, 4, 31-48.

Adrover, R. 1986. Nuevas faunas de roedores en el MioPlioceno continental de la región de Teruel (España). Interés bioestratigráfico y paleoecológico. Instituto de Estudios Turolenses, Teruel, $423 \mathrm{pp}$.

Adrover, R., Mein, P. y Moissenet, E. 1978. Nuevos datos sobre la edad de las formaciones continentales neógenas de los alrededores de Teruel. Estudios geológicos, 34, 205-214.

Ballmann, P. 1969. Die Vögel aus der altburdigalen Spaltenf, llung von Wintershof (West) bei Eichstätt in Bayern. Zitteliana, 1, 5-60.

Bruijn, H. de, Daams, R., Daxner-Höck, G., Fahlsbusch, V., Ginsburg, L., Mein, P. and Morales, J. 1992. Report of the RCMNS working group on fossil mammals, Reisensburg 1990. Newsletters on Stratigraphy, 26 (2/3), 65-118.

Cheneval, J. 1995. Miocene avian localities of France. Acta Universitatis Carolinae, Geologica, 39, 599-611.

Feduccia, A. and Olson, S.L. 1982. Morphological similarities between the Menurae and the Rhino. U, relict passerine birds of the Southern Hemisphere. Smithsonian Contributions to Zoology, 366, 1-22.

Harrison, C.J.O. 1979. Small non-passerine birds of the Lower Tertiary as exploiters of ecological niches now occupied by passerines. Nature, 281, 562-563.

Hoyos, M., Aguirre, E., Morales, J., Pérez González, A. y Soria, M.D. 1974. Parameras de Molina. Cuenca del Jalón, Libro-Guía. Coloquio Internacional sobre Bioestratigrafía Continental del Neógeno Superior y Cuaternario Inferior, 13-48.

Kretzoi, M. 1985. Sketch of the biochronology of the late Cenozoic in Central Europe. Problems of the Neogene and Quaternary in the Carpathian Basin. Akadémiai Kiadó, Budapest, 3-20.

Mlíkovský, J. 1992. The present state of knowledge of the Tertiary birds of Central Europe. Natural History Museum of Los Angeles County (Science Series), 36, 433-458.

Mlíkovský, J. 1995. Tertiary avian faunas of Europe. Acta Universitatis Carolinae, Geologica, 39, 777-818.

Morales, J., Capitán, J., Calvo, J. P. y Sesé, C. 1992. Nuevo yacimiento de vertebrados del Mioceno Superior al Sur de Madrid (Cerro Batallones, Torrejón de Velasco). Geogaceta, 12, 77-80.

Mourer-Chauviré, C. 1995. Paleogene avian localities of France. Acta Universitatis Carolinae, Geologica, 39, 567-598. 
Mourer-Chauviré, C. et Sánchez Marco, A. 1988. Présence de Tyto balearica (Aves, Strigiformes) dans des gisements continentaux du Pliocène de France et d'Espagne. Geobios, 21, 639-644.

Mourer-Chauviré, C., Hugueney, M. et Jonet, P. 1989. Découverte de Passeriformes dans l'Oligocène supérieur de France. Comptes Rendus Hebdomadaires des Scéances de l'Académie des Sciences (Paris) (II), 309. 843-849.

Olson, S.L. 1976. Oligocene fossils bearing on the origins of the Todidae and the Momotidae (Aves: Coraciiformes). Smithsonian Contributions to Paleobiology, 27, 111-119.

Olson, S.L. 1985. The fossil record of birds. Avian Biology, 8, 79-252.

Pérez, B. y Soria, D. 1990. Análisis de las comunidades de mamíferos del Plioceno de Layna (Soria) y La Calera (Teruel). Paleontologia i Evolució, 23, 231-238.

Sánchez Marco, A. 1990. A new bustard (Otididae, Aves) from the early Pliocene of Layna (Soria, Spain). Paleontologia i Evolució, 23, 223-229.

Sánchez Marco, A. 1995a. Tertiary avian localities of Portugal. Acta Universitatis Carolinae, Geologica, 39, 699-701.

Sánchez Marco, A. 1995b. Tertiary avian localities of Spain. Acta Universitatis Carolinae, Geologica, 39, 719-732.

Sánchez Marco, A. (en prensa). Paleornitología del Terciario ibérico y balear. Estudios geológicos.

Schmidt-Kittler, N. 1987. European reference levels and correlation tables. Münchner Geowissenschaftliche Abhandlungen (A), 10, 13-19.

Villalta, J. F. de. 1963. Las aves fósiles del Mioceno español. Boletín de la Real Sociedad Española de Historia Natural (Geología), 61, 263-285.

Villalta, J.F. de y Crusafont, M. 1950. Sobre algunas aves fósiles de Cataluña. Notas y Comunicaciones del Instituto Geológico y Minero de España, 20, 143-156.

Manuscrito recibido: 19 de mayo, 1999 Manuscrito aceptado: 7 de septiembre, 1999 